\title{
Distribución y abundancia de peces de la familia Loricariidae (Pleco) y su relación con los peces de interés comercial en los alrededores de la Isla de Ometepe
}

Jorge T. Corea*, Grettel M. Hernández*, Vilma Solis* y Ariel J. Aguilar**

Recibido: febrero de 2014 / Aceptado: julio de 2014

El objetivo del presente estudio fue determinar la distribución y abundancia de peces de la familia Loricariidae (pleco) y su relación con los peces de interés comercial en los alrededores de la Isla de Ometepe, en el periodo de febrero-agosto 2012. La isla se delimitó en dos áreas, noreste y suroeste y se pescó en dos zonas (A y B). En la zona de pesca A se obtuvo un total de 116 plecos, de los cuales 115 se encontraron en las localidades del área noreste, y el contenido estomacal estaba compuesto por algas filamentosas bentónicas, detritus y materia orgánica acompañada de lodo. En la zona de pesca B se capturaron 1,438 peces pertenecientes a 7 familias y 15 especies. El área noreste presentó mayor volumen de captura que el área suroeste con 1001 y 437 peces, respectivamente. La especie más abundante fue Amphilophus citrinellus, seguida de Brycon guatemalensis y Amphilophus labiatus. Se capturaron 16 plecos, correspondiendo al $1.1 \%$ del total de peces capturados. El género Amphilophus obtuvo valores promedios de factor de condición $(K)$ en las áreas noreste y suroeste de $K=4.4 \pm 0.30$ EEM y $K=2.2 \pm 0.35$ EEM, respectivamente. La localidad más diversa fue San José del Sur $(\mathrm{H}=1.545)$ y la menos diversa fue San Marcos $(\mathrm{H}=0.640)$. El resultado del monitoreo diario en la zona B muestra la captura de 532 plecos, correspondiendo 498 peces para el área noreste y 34 para el área suroeste.

Palabras clave: algas / detritos / factor de condición

Laboratorio de Fisiología Animal, Departamento de Biología, Facultad de Ciencia y Tecnología, Universidad Nacional Autónoma de Nicaragua, León (UNAN-León).

** Laboratorio de Fisiología Animal, Departamento de Biología, Facultad de Ciencia y Tecnología, Universidad Nacional Autónoma de Nicaragua, León (UNAN-León). Correo electrónico: ajagui2002@yahoo.com, Teléfono: 00(505) 86238835. 


\section{Introducción}

Pleco es el nombre común que se le da a los peces pertenecientes a la familia Loricariidae, la cual cuenta con aproximadamente 825 especies distribuidas en 83 géneros y 4 subfamilias: Ancistrinae, Hypoptopomatinae, Hypostominae y Loricariidae (Armbruster, 2004; Nelson, 2006). Los plecos son nativos de la cuenca del Amazonas en Sudamérica. Sin embargo, desde su introducción en los cuerpos de agua epicontinentales (ríos, lagos, lagunas y esteros) se han expandido rápidamente a nivel mundial (Mendoza et al., 2007). En Nicaragua se han obtenido evidencias de presencia de plecos en el lago Cocibolca y el río San Juan a través de una encuesta realizada por técnicos del INPESCA a pescadores de la isla de Ometepe (Instituto Nicaragüense de Pesca y Acuicultura [INPESCA], 2008).

Esta presencia de loricáridos podría causar el desplazamiento y reducción poblacional de las especies nativas debido a la competencia por obtención de alimentos y su comportamiento agresivo y territorial (Mendoza et al., 2007; Marenco, 2010), la ingestión incidental de huevos (Hoover, Killgore \& Cofrancesco, 2004; Cohen, 2008), y sus hábitos alimenticios y de anidación que causan resuspensión del sedimento y cambios en el tamaño y distribución de las partículas en el fondo de los cuerpos de agua (Mendoza et al., 2007). Al desplazarse en grandes cardúmenes, cuando se alimentan dañan o arrancan la vegetación, que es a menudo utilizada como fuente de alimento y sitio de anidación o refugio de otras especies. Además, causan daños severos en las redes de pesca (Marenco, 2010).

En trabajos de investigación que analizan distribución y abundancia es necesario realizar estudios del factor de condición en los peces. Así se logra obtener información directa o indirecta sobre crecimiento, madurez, reproducción, nutrición y, por ende, sobre el estado de salud de las poblaciones. Asimismo, se obtienen datos comparativos interpoblacionales (Granado, 1996; Arismendi, Penaluna \& Soto, 2011) que luego pueden ser usados en modelos predictivos del comportamiento o dinámica, tanto de la población como de la comunidad de peces (McCallum, 2000). Conocer tales características en poblaciones de peces que habitan sistemas con baja o nula intervención antrópica es fundamental para predecir potenciales cambios en poblaciones amenazadas o que habitan sistemas perturbados. En este sentido, las relaciones longitud-peso son utilizadas con frecuencia para modelar las tendencias de la biomasa basadas en la talla, y son ampliamente usadas en el manejo de poblaciones de importancia económica (Treer, Sprem, Torcu-Koc, Sun \& Piria, 2008; Agboola \& Anetekhai, 2008).

De todos los lagos de Nicaragua, el lago Cocibolca presenta mayores opciones de aprovechamiento y relevancia ecológica y económica (Gadea, 2003). Debido a esto, ha sido objeto de diversos estudios para conocer sus características físicoquímicas y biológicas, diversidad piscícola y análisis económico de las actividades pesqueras (Instituto de Fomento Nacional [INFONAC], 1971; Gadea, 2003). Hasta la fecha se desconocen las áreas más pobladas por los plecos en los alrededores de la isla de Ometepe. Por consiguiente, los propósitos de este trabajo fueron: caracterizar la distribución y abundancia de plecos en los alrededores de la isla de Ometepe, corroborar sus características alimenticias y conocer el estado de condición de la especie con mayor frecuencia de captura. 


\section{Materiales y métodos}

\section{1. Área de estudio}

El presente trabajo de investigación se realizó en los alrededores de la isla de Ometepe, situada al oeste del lago Cocibolca, con una extensión de $276 \mathrm{~km}^{2}$ y que está formada por la unión de dos volcanes: Concepción y Maderas. En ella se asientan varios poblados, siendo los principales Moyogalpa y Altagracia (Incer Barquero, 1998).

\subsection{Delimitación del área de estudio y tiempos de ejecución}

La isla de Ometepe se delimitó en dos áreas de estudio, noreste (NE) y suroeste (SO), bajo criterios de disponibilidad de transporte local y tiempo para movilizarse dentro de las diferentes localidades. En cada área se hizo una división por zonas con relación a la distancia de pesca: zona litoral, denominada zona A (desde la costa hasta $50 \mathrm{~m}$ dentro del lago) y zona limnética, denominada zona B (al menos $2 \mathrm{~km}$ de distancia de la costa). Las localidades de estudio para la zona de pesca A fueron: en el área NE Palma y San Miguel y en el área SO San Ramón y Moyogalpa. En la zona de pesca B las localidades de estudio fueron: en el área NE Palma, Punta Gorda, San Miguel y San Marcos y en el área SO San Ramón, Mérida, San José del Sur y Moyogalpa (Ilustración 1).

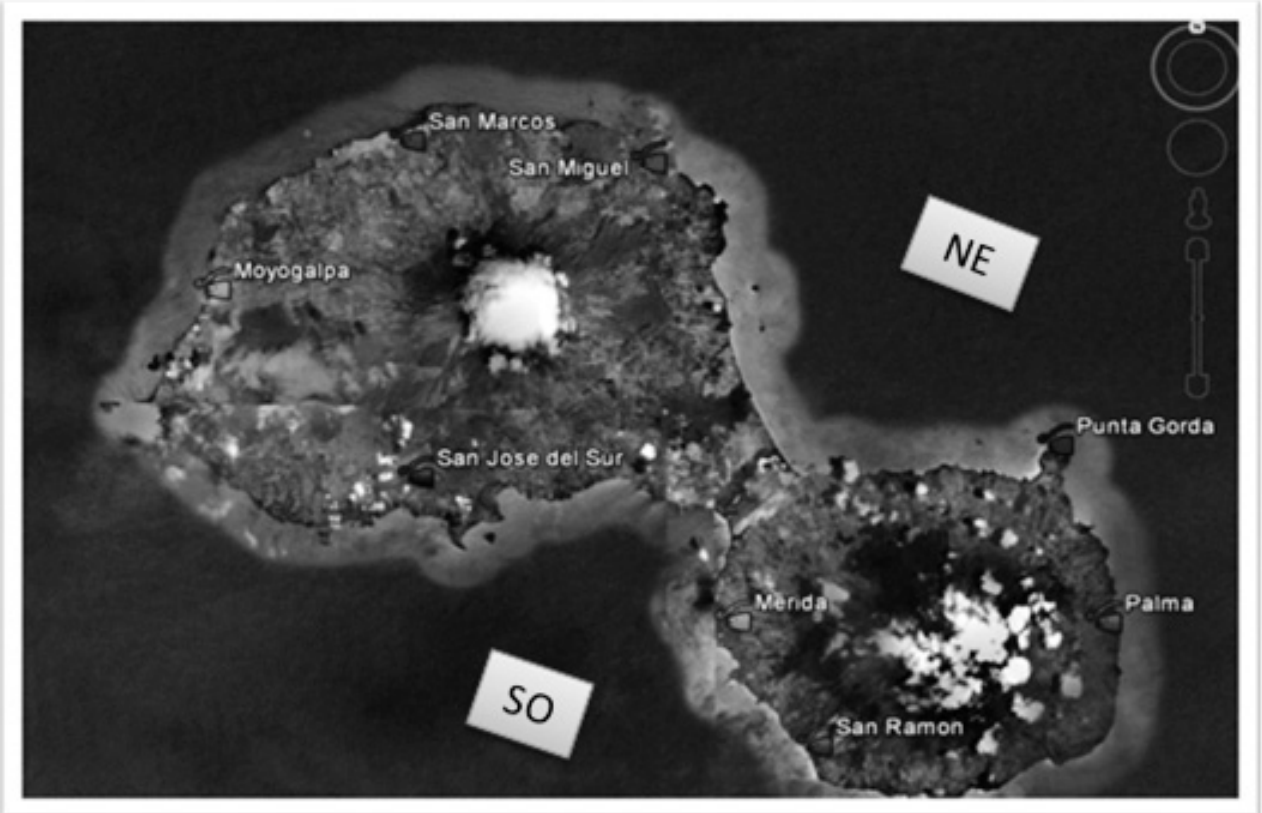

Ilustración 1. Isla de Ometepe. NE: área noreste, SO: área suroeste.

El método de pesca usado para la captura de los peces el de pesca pasiva. En la zona $\mathrm{B}$, debido a su faenar diario, los pescadores mantuvieron colocadas las redes de forma permanente y tomaron los peces capturados en un período de 12 horas de 
puesta de la red (6am - 6pm). Esta situación facilitó la toma de muestras de manera aleatoria una vez al mes y permitió llevar el registro diario de la cantidad de plecos capturados. Todos los peces capturados en cada uno de los puntos de muestreo fueron contados e identificados a nivel de especie según lo propuesto por Bussing (2002) y Villa (1982), y separados por especies para llevar el registro de la captura total en el tiempo. En la zona A, a los plecos se les evaluó contenido estomacal y graso. En la zona $\mathrm{B}$ se trabajó con $\mathrm{N}=15$ peces del género con mayor frecuencia de captura (Amphilophus) para tomar datos sobre longitud total y estándar, y peso del cuerpo.

En la zona A el estudio se llevó a cabo en los meses de julio y agosto, en cambio en la zona B el periodo de estudio correspondió a los meses de febrero a abril, julio y agosto. En ambas zonas se tomaron muestras una vez al mes y, además, por medio de una hoja de registro se llevó registro diario de las capturas de plecos realizadas por los pescadores en la zona $\mathrm{B}$ en los meses de mayo, junio y julio.

\subsection{Análisis de datos}

La diversidad de especies capturadas por los pescadores se calculó con el índice de Shannon y Weaver (1949) a través del método del estimador de ligamiento máximo (ELM). La determinación de la abundancia de peces para cada área se realizó usando la estimación del rendimiento en forma de 'captura por unidad de esfuerzo' (CPUE) expresada en gramos por hora. Se obtuvo siempre una CPUE para cada pesca y se usó este valor como unidad, de tal forma que las diferentes CPUEs estimadas por categoría (zonas, áreas) se determinaron como el promedio de las CPUEs por pesca.

A los peces del género Amphilophus se les evaluó el factor de condición $(K)$ mediante la aplicación de la fórmula recomendada por King (1995) para comparar la condición de los peces entre localidades y áreas de estudio, y usando el programa Sigma Stat (SPSS Inc., Chicago, IL) para determinar la relación talla-peso.

La comparación entre las capturas de peces y las variables antes mencionadas por localidad, zona y entre áreas, se realizó usando Sigma Stat (SPSS Inc., Chicago, IL). Los datos se muestran como la media \pm E.E.M. Las diferencias de todas las variables testadas entre localidades se obtuvieron mediante la evaluación de un ANOVA de una vía y la diferencia entre áreas se evaluó mediante la prueba t-students. Tras los análisis de varianza se realizó el test de comparaciones múltiples de Student Newman Keuls y en todos los casos el nivel de significación se estableció con un valor de $(\mathrm{P}<0.05)$. Previo a estas pruebas estadísticas se analizaron los datos mediante una prueba de normalidad (Shapiro-Wilks) y de homogeneidad de varianzas (prueba C de Cochran).

\section{Resultados y discusión}

Según INPESCA (2008), desde el año 2004 los pescadores de la parte oriental del lago Cocibolca reportaron capturas de una especie de pez desconocido para ellos y que aparecía eventualmente en las capturas en mínimas cantidades. Posteriormente, a partir del año 2007, comenzaron a reportar la presencia de esta especie en 
sectores como Puerto Díaz, San Miguelito, Morrito, San Carlos, San Ramón (isla de Ometepe) y en la parte occidental del lago Cocibolca. Sin embargo, estudios realizados en algunas de las zonas antes mencionadas (Puerto Díaz, Morrito, San Carlos, El Nancital y San Miguelito) en el periodo 2005-2006 no reportaron presencia de plecos (Hernández \& Saborío, 2007).

El análisis sobre las características taxonómicas y biométricas realizado por INPESCA (2008) a este pez lo asemeja con el género Hypostomus, coincidiendo con las características fenotípicas reportadas por Marenco (2010) en el pez pleco capturado en los humedales de Caño Negro, el cual presenta un patrón de manchas definidas en la parte ventral similar al patrón de manchas que presenta el pleco capturado en el lago Cocibolca de Nicaragua. Sin embargo, actualmente no se sabe la especie a la cual pertenece el pleco que invade el ambiente acuático del lago Cocibolca. En consecuencia, se realizó el diseño experimental para obtener evidencias sobre la distribución y abundancia de plecos en los alrededores de la isla de Ometepe, usando como muestra las especies de peces capturados por los pescadores en el área limnética durante su actividad pesquera y en base a las características de esta especie, la captura dirigida al pleco en el área litoral.

La hipótesis consistió en que en las áreas menos alejadas de la costa, que presentan características semejantes a las señaladas por Marenco (2010) y Mendoza et al. (2009), como son: poca profundidad, suelos sedimentosos, alta cantidad de perifiton, relieve acuático rocoso, tocones sumergidos, plantas acuáticas y alta cantidad de detritos, es de esperarse encontrar presencia de plecos. Por el contrario, si en algún área las condiciones antes mencionadas no están presentes, esperaríamos que los resultados denoten poco o nada la presencia de plecos.

\subsection{Distribución - abundancia y aspectos biológicos de los plecos en zona litoral}

Se sabe que los plecos prefieren hábitats rocosos con abundantes desechos leñosos debido a que su alimentación está basada fundamentalmente en algas bentónicas. También requieren de aguas poco profundas que garanticen condiciones óptimas para anidar, debido a que estos peces cavan sus galerías a orilla de la costa (Marenco, 2010; Mendoza et al., 2007; Liang, Wu \& Shieh, 2005; Page \& Robbins, 2006). El área noreste (NE) de la isla de Ometepe presenta características similares a las antes mencionadas debido a la presencia de los grandes islotes que caracterizan al gran lago Cocibolca (Incer Barquero, 1998). Esta puede ser la causa por la que en nuestros resultados las localidades del área NE (San Miguel y Palma) presenten mayor presencia de plecos que las localidades del área suroeste (SO) (San Ramón y Moyogalpa) (Ilustración 2). 


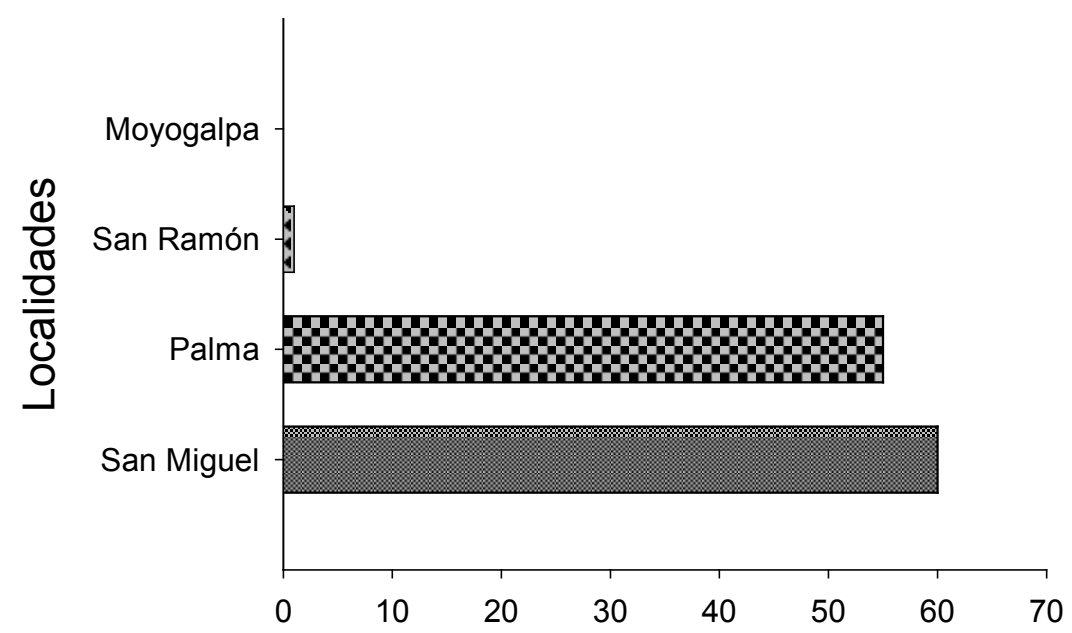

Ilustración 2. Captura total de plecos en zona limnética (zona A) en los alrededores de la isla de Ometepe.

En ese sentido, nuestros resultados coinciden con los de estudios similares realizados en otras regiones del mundo, donde las áreas de estudio presentan condiciones análogas al área NE de la isla de Ometepe (Wakida-Kusunoki \& Amadordel Ángel, 2011). Pero considerando la relación pleco-condiciones del entorno, resulta contradictoria la poca captura de plecos en la localidad de San Ramón - área SO (Ilustración 2), donde el litoral es rocoso con presencia de abundante materia orgánica, características orográficas y relieve óptimo para su desarrollo.

Al realizar el estudio del contenido estomacal en los plecos se observó que la especie posee un sistema digestivo de estructura tubular y un tracto gastrointestinal sumamente alargado. Una gran cantidad de grasa envuelve al sistema digestivo, de tal forma que al diseccionar los organismos por su cavidad abdominal se logra apreciar un abundante líquido amarillo aceitoso. Se observó que la materia alimentaria dentro del tracto digestivo estaba compuesta por algas filamentosas bentónicas, detritus y materia orgánica acompañada de abundante lodo, lo cual coincide con estudios anteriores (Gestring, 2006; Yossa \& Araujo Lima, 1998; Donovan \& Bittong, 2009).

\subsection{Captura - distribución - abundancia y aspectos biológicos de los peces capturados en la zona limnética (zona B)}

A fin de corroborar las evidencias iniciales presentadas por los pescadores y el reporte de los técnicos de INPESCA (2008) sobre la presencia de plecos en los sitios de pesca, diseñamos un método de muestreo que duplica el número de puntos de toma de muestras con respecto al diseño ejecutado en la zona litoral. Lo hicimos no solamente para evaluar la distribución de los plecos sino también para determinar el porcentaje de los mismos con respecto a todas las especies de peces capturados por los pescadores en su faenar diario. Los resultados obtenidos muestran que los pescadores del área NE en promedio obtienen mayor captura de peces que los pescadores del área SO de la isla de Ometepe (véase Ilustración 3). 


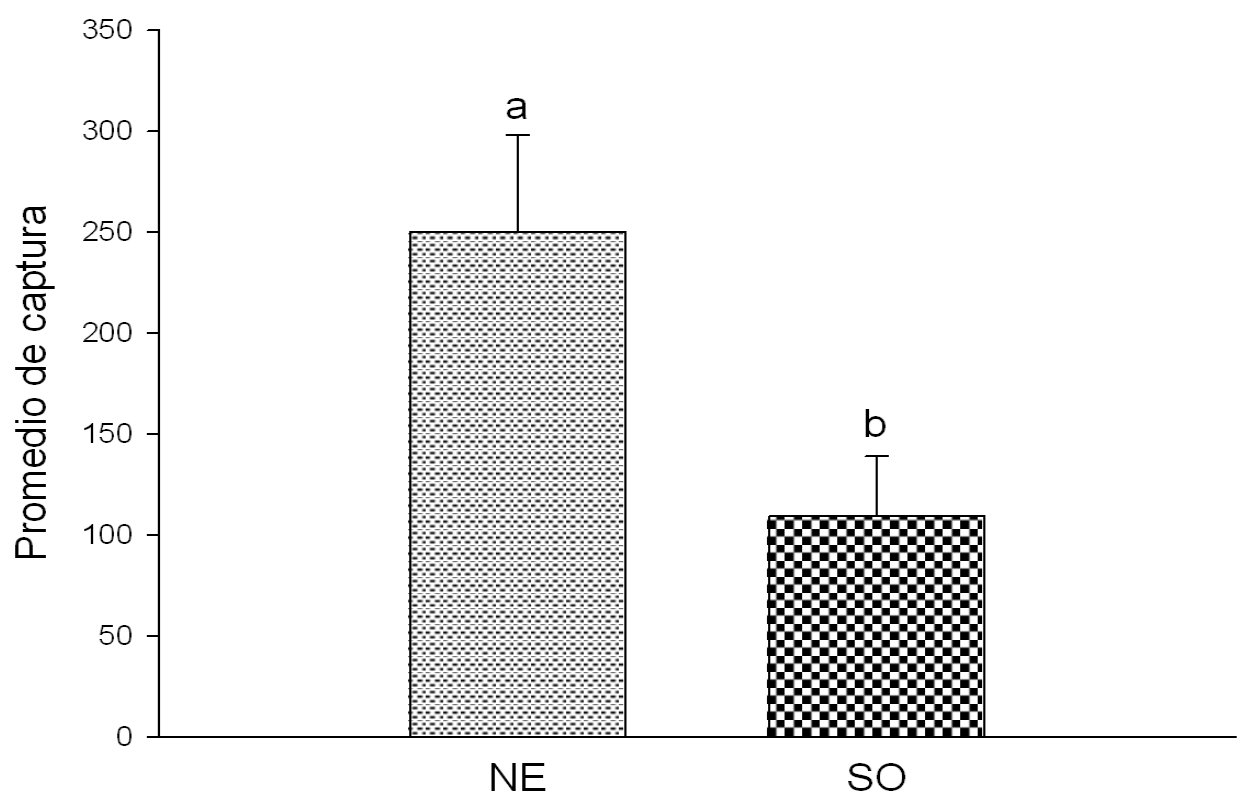

Ilustración 3. Promedio de peces capturados en los cinco meses de estudio en los alrededores de la isla de Ometepe

Nota: zona noreste (NE) y zona suroeste (SE). Cada valor se corresponde a la media \pm EEM. Letras diferentes indican diferencias significativas $(P<0.05)$ entre áreas.

No obstante, debido a que nuestro estudio se focalizó en la distribución y abundancia de peces, no evaluamos el estado nutricional ni los factores ambientales en el cuerpo de agua, información que nos permitiría discutir con más claridad la causa de este fenómeno. Sin embargo, adelante mostraremos algunas evidencias que permitirán ampliar la discusión con más razonabilidad.

Partiendo de que el área NE presenta mayor promedio de captura que el área $\mathrm{SO}$, en los cinco meses de estudio evaluamos los promedios de captura en todas las localidades de las dos áreas muestreadas. Lo más relevante es que Punta Gorda es la localidad que presenta mayor promedio de captura de todas las localidades y difiere significativamente de las localidades de su área (Ilustración 4). Estos resultados indican que en ese sector el cuerpo de agua podría presentar condiciones óptimas para la vida piscícola, similares a las encontradas en otras zonas, donde los ambientes acuáticos presentan alto contenido nutritivo que provoca el crecimiento de plantas, invertebrados y microorganismos que garantizan abundante alimento a los peces (Lowe-McConnell, 1975; Saint Paul et al., 2000; Rahim et al., 2009). 


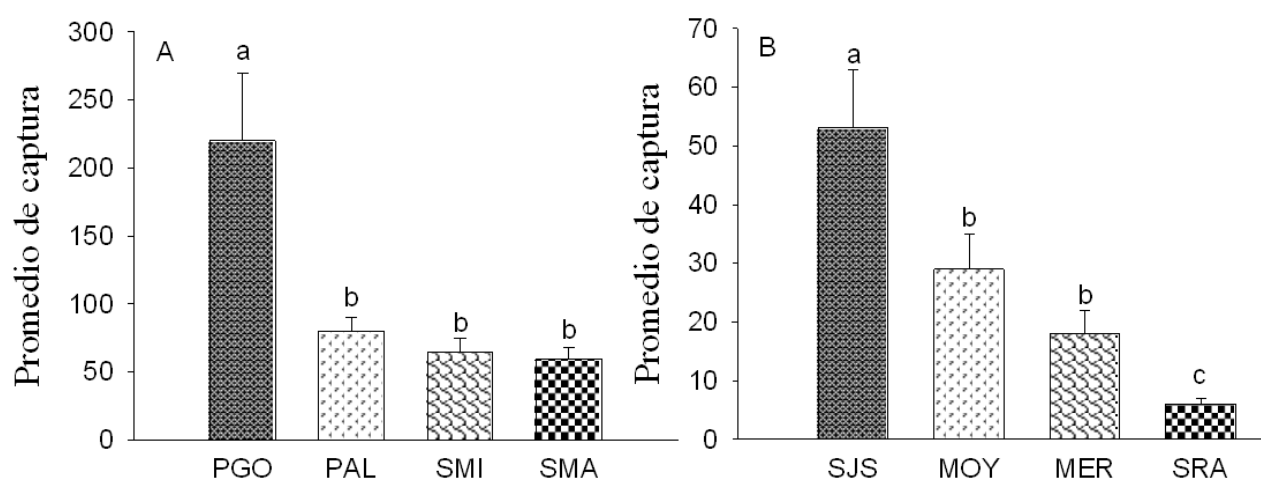

Ilustración 4. Promedio de peces capturados en los cinco meses de estudio en los alrededores de la isla de Ometepe

Nota: A) zona noreste y B) zona suroeste. Las localidades son: Punta gorda (PGO), Palma (PAL), San Miguel (SMI), San Marcos (SMA), San José del Sur (SJS), Moyogalpa (MOY), Mérida (MER) y San Ramón (SRA).Cada valor se corresponde a la media \pm EEM. Letras diferentes indican diferencias significativas $(P<0.05)$.

En total se capturaron 1,438 peces distribuidos en 15 especies. Los plecos corresponden al $1.11 \%$ del total (Cuadro 1). Asimismo, todas las especies que encontramos han sido reportadas en anteriores estudios realizados en el lago Cocibolca (Gadea, 2003; Hernández \& Saborío, 2007). Considerando que nuestro estudio evaluó solamente los peces capturados por los pescadores en su faenar diario, con principal interés en los plecos, es importante señalar que los pescadores tenían objetivos definidos hacia la captura de determinadas especies de interés comercial, situación que incidía en la puesta de la red en puntos con batimetría menor o mayor dentro del cuerpo de agua. Esta situación puede ser la causa de que nuestros resultados solamente reporten 15 especies de peces. Por tanto, estos resultados no permiten descartar la presencia de las otras especies de peces reportadas anteriormente en el lago Cocibolca (INFONAC, 1971; Gadea, 2003; Hernández \& Saborío, 2007). 
Cuadro 1. Cantidad de individuos, por especie, capturados por los pescadores en los alrededores de la isla de Ometepe

Nota: Periodo de febrero-abril y julio-agosto, 2012. Las localidades se corresponden con: Palma (PAL), Punta Gorda (PGO), San Marcos (SMA), San Miguel (SMI), Mérida (MER), Moyogalpa (MOY), San José del Sur (SJS) y San Ramón (SRA).

\begin{tabular}{|l|l|l|l|l|l|l|l|l|}
\hline \multirow{2}{*}{ Especies } & \multicolumn{3}{|c|}{ Área Noreste } & \multicolumn{5}{c|}{ Área Suroeste } \\
\cline { 2 - 10 } & PAL & PGO & SMA & SMI & MER & MOY & SJS & SRA \\
\hline Amphilophus citrinellus & 182 & 260 & - & 103 & 24 & 98 & 88 & 14 \\
\hline Amphilophus labiatus & 35 & - & 78 & - & - & - & 2 & 3 \\
\hline Astatheros longimanus & - & 4 & - & - & - & 8 & 20 & - \\
\hline Astatheros rostratus & - & 14 & - & - & 5 & 21 & 72 & 1 \\
\hline Brycon guatemalensis & 15 & 104 & 40 & 40 & 3 & 1 & 4 & 3 \\
\hline Centropomus pectinatus & - & - & - & - & - & 2 & - & - \\
\hline $\begin{array}{l}\text { Cichlasoma } \\
\text { maculicauda }\end{array}$ & - & 13 & - & - & - & - & - & - \\
\hline Dorosoma chavesi & - & 22 & - & - & - & - & 16 & - \\
\hline Gobiomorus dormitory & - & - & - & - & 5 & 5 & 4 & - \\
\hline $\begin{array}{l}\text { Hypsophrys } \\
\text { nicaraguensis }\end{array}$ & 22 & 27 & - & 10 & - & 19 & 3 & - \\
\hline Loricariidae & 13 & - & - & 2 & - & - & - & 1 \\
\hline Oreochromis niloticus & - & - & - & - & - & 1 & - & - \\
\hline Parachromis dovii & 7 & 10 & - & - & - & - & - & - \\
\hline $\begin{array}{l}\text { Parachromis } \\
\text { managuensis }\end{array}$ & - & - & - & - & - & - & 2 & 2 \\
\hline Pomadasys croco & - & - & - & - & 1 & 2 & 7 & - \\
\hline Total & 274 & 454 & 118 & 155 & 38 & 157 & 218 & 24 \\
\hline
\end{tabular}

Continuando con el análisis de estos resultados observamos que Punta Gorda y San José del Sur presentan el mayor índice de diversidad (Cuadro 2) y promedio de captura en sus respectivas áreas (Ilustración 4). Se ha observado que los peces, incluidos los plecos, presentan comportamiento migratorio que los dirige hacia sitios con mayor cantidad de alimento y condiciones de anidación o reproducción (Ocaña $\&$ Lot, 1996). Por tanto, es probable que los peces que habitan los alrededores de la isla de Ometepe realicen las mismas acciones o que la diferencia en la cantidad de individuos capturados en las dos áreas de estudio (NE y SO) pudiera deberse a la selectividad del arte de pesca, la selección del sitio de captura o la temporada de pesca. 
Cuadro 2. Índice de diversidad de Shannon-Wiener usando la captura total de peces en la zona B, en los alrededores de la isla de Ometepe.

Nota: Noreste (NE), suroeste (SO), Punta Gorda (PGO), Palma (PAL), San Miguel (SMI), San Marcos (SMA), San José del Sur (SJS), San Ramón (SRA), Moyogalpa (MOY) y Mérida (MER).

\begin{tabular}{|l|l|l|}
\hline \multicolumn{1}{|c|}{ Área } & Localidad & \multicolumn{1}{c|}{$\begin{array}{c}\text { Índice de Shannon-Wiener } \pm \\
\text { E.E }\end{array}$} \\
\hline NE & PGO & $\begin{array}{l}1.413 \\
(0.052)\end{array}$ \\
\hline NE & PAL & $\begin{array}{l}1.134 \\
(0.064)\end{array}$ \\
\hline NE & SMI & $\begin{array}{l}0.854 \\
(0.061)\end{array}$ \\
\hline NE & SMA & $\begin{array}{l}0.640 \\
(0.029)\end{array}$ \\
\hline SO & SJS & $\begin{array}{l}1.545 \\
(0.068)\end{array}$ \\
\hline SO & SRA & $\begin{array}{l}1.306 \\
(0.194)\end{array}$ \\
\hline SO & MOY & $\begin{array}{l}1.256 \\
(0.090)\end{array}$ \\
\hline SO & MER & $\begin{array}{l}1.120 \\
(0.147)\end{array}$ \\
\hline
\end{tabular}

La abundancia de las especies se evaluó en base a la frecuencia de captura por unidad de esfuerzo (CPUE). En orden decreciente las tres especies más abundantes son: Amphilophus citrinellus, Brycon guatemalensis y Amphilophus labiatus (Ilustración 5). No obstante, a excepción de los individuos de la familia Loricariiidae, las especies identificadas coinciden en aparición con estudios anteriores (Hernández \& Saborío, 2007; Gadea, 2003).

Nuestros resultados coinciden con Bussing (2002), Hernández y Saborío (2007) y Gadea (2003) en que Brycon guatemalensis y Amphilophus citrinellus son las especies más abundantes. Por otro lado, en contraste con nuestros resultados, Atractosteus tropicus, que Gadea (2003) reporta en tercer lugar de importancia y que en el estudio de Hernández y Saborío (2007) representa un 1\% de frecuencia de apariciones, no fue capturada durante el periodo de muestreo en los alrededores de la isla de Ometepe. Esto se debe probablemente a la distribución de la especie en el lago Cocibolca, a la dirección de las capturas realizadas por los pescadores o a la posible disminución poblacional de la especie.

La relación longitud-peso y el factor de condición $(K)$ son descriptores de gran interés en la biología de poblaciones de peces, ya que aportan información fundamental sobre estrategias de crecimiento, estado nutricional y reproductivo. También son parámetros ampliamente utilizados para comparar la condición de las poblaciones que habitan en sistemas acuáticos con distintos grados de intervención 


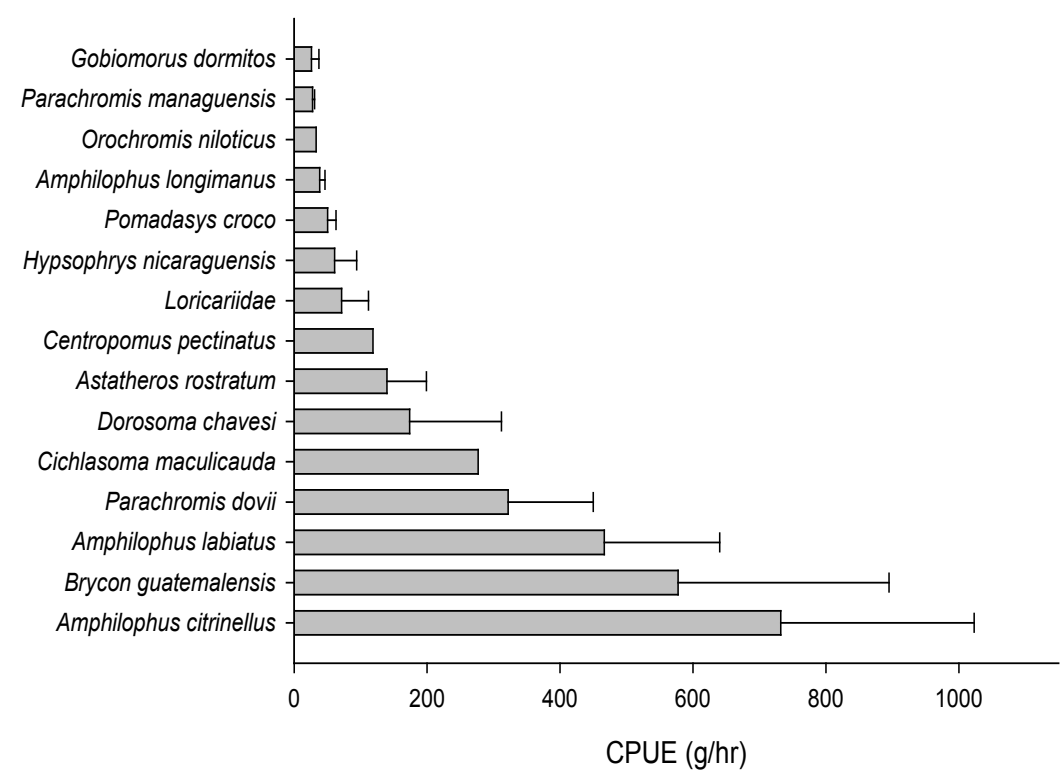

Ilustración 5. Promedio y desviación estándar del rendimientos por lance de las especies más importantes

antrópica (Cifuentes et al., 2012). En ese sentido, para comparar el estado nutricional de los peces en ambas áreas de estudio, evaluamos el factor de condición $(K)$ del género de pez con más frecuencia de captura (Amphilophus). Los resultados muestran diferencia de los valores de $K$ entre áreas (Ilustración 6), lo cual es interesante debido a que se sabe que en los peces, como en otros vertebrados, el comportamiento alimentario está regulado por factores medioambientales y por complejos mecanismos homeostáticos que involucran factores hormonales periféricos y centrales, y niveles de metabolitos presentes en la sangre (Aguilar, Conde-Sieira, Polakof, Miguez \& Soengas, 2010; Aguilar, Conde-Sieira, López-Patino, Miguez \& Soengas, 2011; Volkoff, Unniappan \& Kelly, 2009). Asimismo, el principal regulador de la ingesta de alimento es el contenido energético presente en el alimento debido a que los peces comen exclusivamente hasta alcanzar un estatus energético óptimo (Boujard \& Médale, 1994; Morales, Cardenete, De la Higuera \& Sanz, 1994). Es más, a pesar de que los peces han desarrollado diferentes hábitos alimenticios, está demostrado que todos ellos comen hasta alcanzar la saciedad (Dabrowski, 1993; Raubenheimer, Simpson \& Mayntz, 2009).

Por tanto, es de esperarse que los peces del género Amphilophus presenten iguales valores de $K$ en ambas áreas, siempre y cuando la disponibilidad de alimento y el contenido nutricional del mismo estén presentes en el cuerpo de agua. En contraste, nuestros resultados muestran que los peces del área NE presentan diferencias en los valores de $K$ con respecto a los peces del área SO, lo que sugiere que algunos factores ambientales podrían estar estresando o incidiendo en la ingestametabolismo de los peces (Volkoff et al., 2009), no permitiéndoles alcanzar un estado optimo de K. Pero para esclarecer este fenómeno es necesario realizar un estudio completo de la salud del cuerpo de agua del lago Cocibolca. Bajo ese contexto, la mayor captura de peces en el área NE (Ilustración 3) podría indicar que el área SO no presenta condiciones óptimas para el bienestar animal, obligando a los peces a 


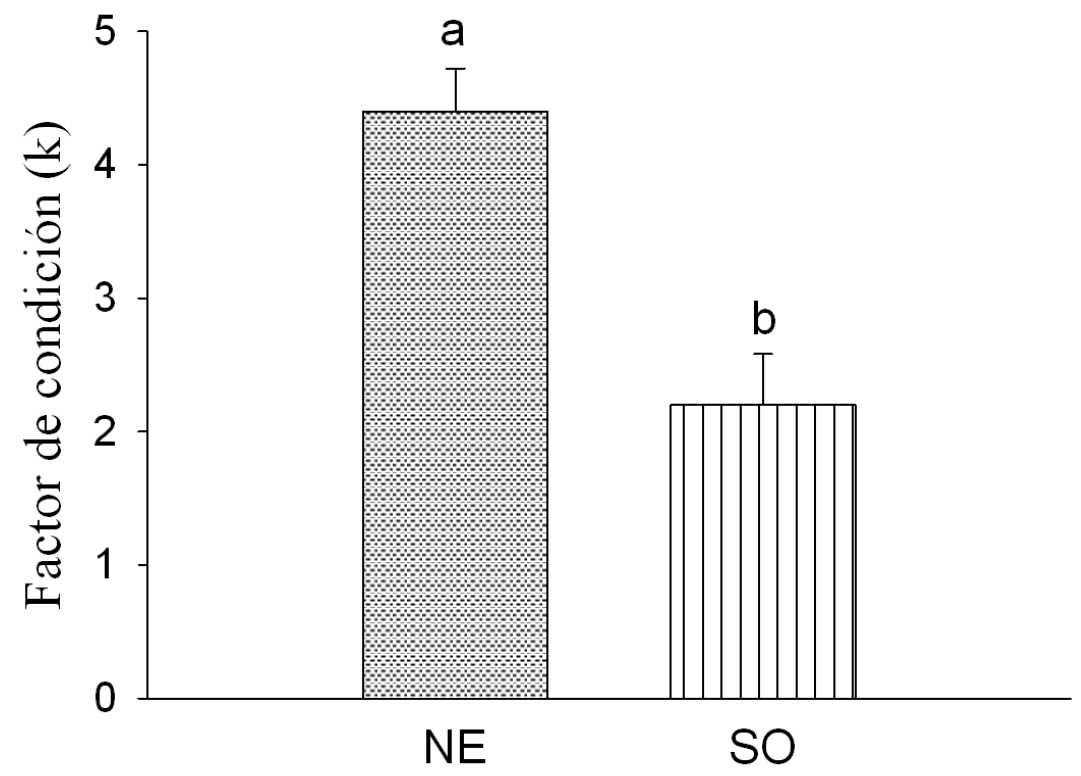

Ilustración 6. Valores promedios de índice de factor de condición (k) en los cinco meses de estudio para el área noreste (NE) y el área suroeste (SO). Nota: Cada valor se corresponde a la media \pm EEM. Letras diferentes indican diferencias significativas $(P<0.05)$ entre áreas.

migrar del área SO al área NE. Además, otro factor que podría estar incidiendo en la poca captura de peces en el área $\mathrm{SO}$ es la presencia de barcos de pesca comercial provenientes de la ciudad de Granada (Cooperativa de Pesca Artezanal "San José del Sur”, comunicación personal).

\subsection{Monitoreo de plecos en la zona B}

Producto del resultado de la toma de datos diarios por los pescadores, el monitoreo de plecos capturados en la zona B presenta similares resultados a los encontrados en la zona A, en la cual de manera general los mayores promedios de captura se observan en el área NE (Ilustración 7). Como se mencionó anteriormente, el área $\mathrm{NE}$ presenta características orográficas y de vegetación diferente al área SO. En consecuencia, es probable que los plecos migren hacia sitios con una alta diversidad de plantas acuáticas que garanticen mejor condición para su desarrollo (Ocaña \& Lot, 1996).

La presencia de plecos en el lago Cocibolca de Nicaragua es una amenaza para la biodiversidad de especies de peces nativos del sector debido a que está demostrado que una cantidad pequeña de plecos puede disminuir de manera considerable la abundancia de la vegetación acuática y piscícola en cualquier cuerpo de agua (Power, 1990). Por tanto, es necesario tomar medidas que conlleven a controlar el esparcimiento de las poblaciones de esta especie mediante diversos usos que se les puede dar a los plecos. Uno de ellos puede ser la elaboración de harina de pleco para alimento de tilapias (Arroyo, 2008). 


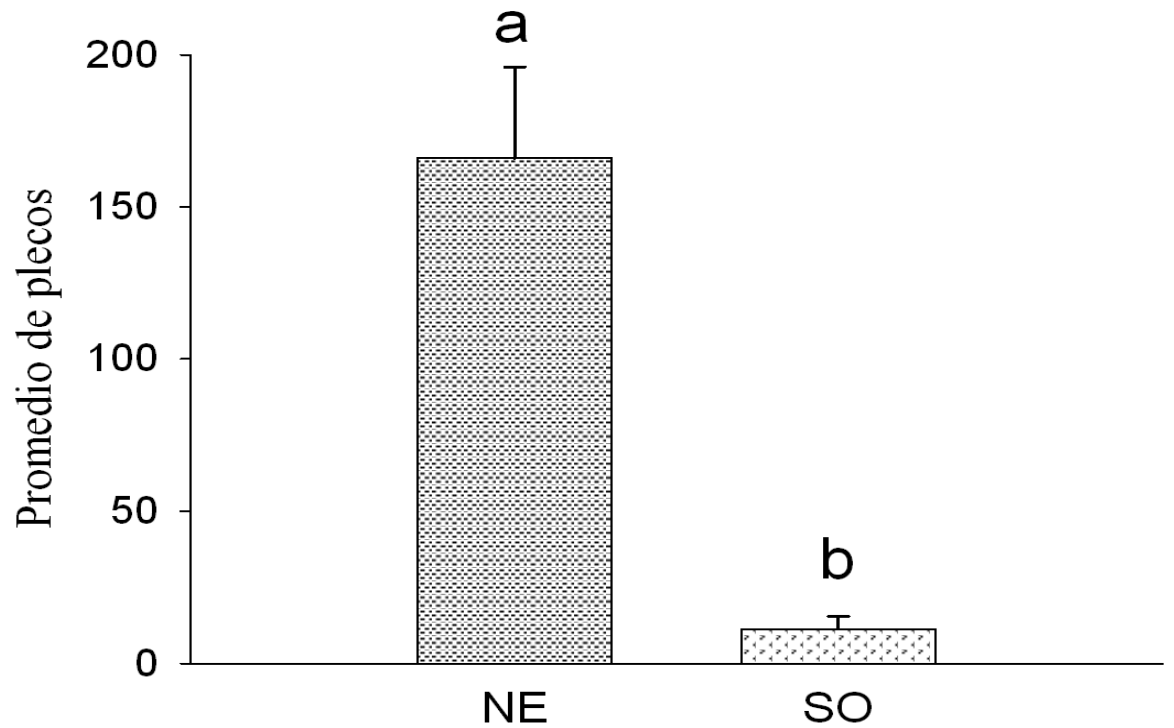

Ilustración 7. Valores promedios de monitoreo de plecos para el área noreste (NE) y el área suroeste $(\mathrm{SO})$

Nota: Cada valor se corresponde a la media \pm EEM. Letras diferentes indican diferencias significativas $(P<0.05)$ entre áreas.

\section{Agradecimientos}

El presente trabajo de investigación ha sido realizado gracias a la Xunta de GaliciaEspaña por medio de una subvención a la Asociación Amigos de la Tierra España para el desarrollo del proyecto "Fortalecimiento de grupos organizados para la Pesca Artesanal Sostenible y la conservación de los recursos naturales en la Isla de Ometepe, Fase II".

El desarrollo experimental fue apoyado por: Laboratorio de Investigaciones Marinas y Acuícolas de la UNAN-León (LIMA), Vicerrectorías de la UNAN-León (Relaciones Externas e Investigación, Postgrado y Proyección Social), Fundación entre Volcanes (FEV), Alcaldía de Moyogalpa, Alcaldía de Altagracia y Cooperativas de pescadores artesanales de la isla de Ometepe. 


\section{Referencias bibliográficas}

Agboola, J. I. \& Anetekhai, M. A. (2008). Length-weight relationships of some fresh and brackish water fishes in Badagry Creek, Nigeria. Journal Of Application Ichthyology, (24), 623-625.

Aguilar, A. J., Conde-Sieira, M., López-Patino, M. A., Miguez, J. M. \& Soengas, J. L. (2011). In vitro leptin treatment of rainbow trout hypothalamus and hindbrain affects glucosensing and gene expression of neuropeptides involved in food intake regulation. Peptides, (32), 232-240.

Aguilar, A.J., Conde-Sieira, M., Polakof, S., Miguez, J. M., Soengas, J. L. (2010). Central leptin treatment modulates brain glucosensing function and peripheral energy metabolism of rainbow trout. Peptides, (31), 1044-1054.

Arismendi, I., Penaluna, B. \& Soto, D. (2011). Body condition indices as a rapid assessment of the abundance of introduced salmonids in oligotrophic lakes of southern Chile. Lake and Reservoir Management, (27), 61-69.

Armbruster, J. W. (2004). Phylogenetic relationships of the suckermouth armored catfishes (Loricariidae) with emphasis on the Hypostominae and the Ancistrinae. Zoological Journal of the Linnean Society, (141), 1-80.

Arroyo, M. (2008). Aprovechamiento de la harina de Plecostomus spp. como ingrediente en alimento para el crecimiento de tilapias (Oreochromis niloticus). Tesis de maestría no publicada. Instituto Politécnico Nacional, Mexico.

Boujard, T. \& Médale, F. (1994). Regulation of voluntary feed intake in juvenile rainbow trout fed by hand or by self feeders with diets containing 2 different protein energy ratios. Aquat. Living Resour., 7, 211-215.

Bussing, W. A., (2002). Peces de las aguas continentales de Costa Rica. San José: Universidad de Costa Rica.

Cifuentes, R., González, J., Montoya, G., Jara, A., Ortíz, N., Piedra, P. \& Habit, E. (2012). Relación longitud-peso y factor de condición de los peces nativos del río San Pedro (cuenca del río Valdivia, Chile). Gayana, 75 (2), 101-110.

Cohen, K. L. (2008). Gut content and stable isotope analysis of exotic suckermouth catfishes (Hypostomus) in the San Marcos River, Texas: A concern for spring endemics?. Tesis de maestría no publicada. San Marcos, Texas State University.

Dabrowski, K. (1993). Ecophysiological adaptations exist in nutrient requirements of fish: true or false? Comp. Biochem. Physiol. A., 104, 579-584.

Donovan, G. \& Bittong, R. A. (2009). Digestive enzyme activities and gastrointestinal fermentation in wood-eating catfishes. J Comp Physiol B., 179, 1025-1042.

Gadea, E. V. (2003). Estudio Biológico pesquero de las especies comerciales en San Carlos y Solentiname. Rio San Juan, Nicaragua. Managua: Asociación de Cooperación Rural en África y América Latina (ACRA).

Gestring, K. (2006). Shoreline Erosion Assessment of Loricariidae Catfishes in Florida. Boca Ratón, Florida: Florida Fish and Wildlife Conservation Commission.

Granado, C. (1996). Ecología de peces. Sevilla, España: Secretariado de Publicaciones de la Universidad de Sevilla.

Hernández, P. \& Saborío, R. (2007). Abundancia relativa de los peces en la costa oriental del lago de Nicaragua. (1 ed). Managua: INPESCA. 
Hoover, J. J., Killgore, K. J. \& Cofrancesco, A. F. (2004). Suckermouth Catfishes: Threats to Aquatic Ecosystems of the United States. Aquatics Nuisance Species Research Program (ANSRP), 04-1.

Incer Barquero, J. (1998). Geografía básica de Nicaragua. Managua: Editorial Hispamer. Instituto de Fomento Nacional [INFONAC]. (1971). Los recursos pesqueros del Lago de Nicaragua. Boletín Nicaragüense de Bibliografía y Documentación. EneroMarzo. Managua: Autor.

Instituto Nicaragüense de Pesca y Acuicultura [INPESCA]. (2008). Revisión preliminar para la identificación de la especie de pez exótico reportado recientemente en el lago Cocibolca de Nicaragua. Managua: Autor.

King, M. (1995). Fisheries biology, assessment and management. Fishing News Books, Oxford, Inglaterra: Blackwell Science.

Liang, S. H., Wu, H. P. \& Shieh, B. S. (2005). Size structure, reproductive phenology, and sex ratio of an exotic armored catfish (Liposarcus multiradiatus) in the Kaoping River of southern Taiwan. Zoological Studies, 44, 252-259.

Lowe-McConnell, R. H. (1975). Ecological aspect of seasonality in fishes of tropical water. Symposia of the Zoological Society of London, 44, 219-241.

Marenco, Y. (2010). El Pez Diablo: una especie exótica invasora. Biocenosis, 23 (2), $16-19$.

Mccallum, H. (2000). Population Parameters: Estimation for Ecological Models. London: Blackwell Science Ltd. Oxford.

Mendoza, R., Fisher, J., Courtenay, W., Ramírez, C., Orbe, A., Escalera, C., Álvarez, P., Koleff, P. \& Contreras, S. (2009). Evaluación trinacional de riesgos de los plecos (Locariidae). En: Comisión para la Cooperación Ambiental. (Eds.). Directrices trinacionales para la evaluación de riesgos de las especies acuáticas exóticas invasivas. (pp. 17-29). Montreal: Comisión para la Cooperación Ambiental.

Mendoza, R., Contreras, S., Ramírez, C., Koleff, P., Álvarez, P. \& Aguilar, V. (2007). Los peces diablo: Especies invasoras de alto impacto. CONABIO. Biodersitas, $70,15$.

Morales, A. E., Cardenete, G., De la Higuera, M. \& Sanz, A. (1994). Effects of dietary protein source on growth, feed conversion and energy utilization in rainbow trout, Oncorhynchus mykiss. Aquaculture, 124, 117-126.

Nelson, J. S. (2006). Fishes of the world. (4ta. Ed.). Hoboken, Nueva Jersey: John Wiley and Sons.

Ocaña, D. \& Lot, A. (1996). Estudio de la vegetación acuática vascular del sistema fluvio-laguna-deltáico del río Palizada, en Campeche, México. Anales del Instituto de Biología. Universidad Nacional Autónoma de México. Serie Botánica, 67, 303-327.

Page, L. M. \& Robins, R. H. (2006). Identification of sailfin catfishes (Teleostei: Loricariidae) in Southern Asia. The Raffles Bulletin of Zoology, 54 (2), 455-457.

Power, M. E. (1990). Resource Enhancement by indirect effects of grazers: Armored catfish, algae, and sediment. Ecology, 71, 897-904.

Rahim, K. A. A., Daud, S., Siraj, S., Arshad, A., Esa, Y. \& Ibrahim, E. (2009). Freshwater fish diversity and composition in Batang Kerang floodplain, Balai Ringin, Sarawak. Pertanika. Journal of Tropical Agriculture Science, 32, 7-16. 
Raubenheimer, D., Simpson, S. J. \& Mayntz, D. (2009). Nutrition, ecology and nutritional ecology: toward an integrated framework. Func. Ecol., 23, 4-16.

Saint-Paul, U., Zuanon, J., Villacorta Correa, M.A., García, M., Fabré, N., Berger, U. \& Junk, W. J. (2000). Fish communities in central Amazonian white and blackwater floodplains. Environmental Biology of Fishes, 57, 235-250.

Shannon, C. E. \& Weaver, W. (1949). The Mathematical Theory of Communication. Urbana, Illinois: University Illinois Press.

Treer, T., Sprem, N., Torcu-Koc, H., Sun, Y. \& Piria, M. (2008). Length-weight relationships of freshwater fishes of Croatia. Journal of Application Ichthyology, 24, 626-628.

Villa, J. (1982). Peces nicaragüenses de agua dulce. Managua: Fondo de Promoción Cultural, Banco de América.

Volkoff, H., Unniappan, S. \& Kelly, S. P. (2009). The endocrine regulation of food intake. In: Bernier, N. J., Van der Kraak, G., Farrell, A. P., Brauner, C.J. (Eds.). Fish neuroendocrinology. (pp. 421-465). Amsterdam: Academic Press.

Wakida Kusunoki, A. T. \& Amador del Ángel, L. E. (2011). Aspectos biológicos del pleco invasor Pterygoplichthys pardalis (Teleostei: Loricariidae) en el rio Palizada, Campeche, Mexico. Revista Mexicana de Biodiversidad, 82 (3), 870-878.

Yossa, M. I. \& Araujo Lima, C. A. (1998). Detritivory in two Amazonian fish species. Journal of Fish Biology, 52 (6), 1141-1153. 